T. А. Машорипова, А. А. Гришкевич

Белорусский государственный технологический университет

\title{
ОСОБЕННОСТИ ТРАНСФОРМАЦИИ УГЛА РЕЗАНИЯ СВЕРЛИЛЬНОГО ИНСТРУМЕНТА В ЗАВИСИМОСТИ ОТ ПОЛОЖЕНИЯ ЕГО ЛЕЗВИЯ
}

Статья описывает теоретическое исследование влияния угла наклона лезвия к оси винтового сверла на угол резания. В данной работе установлена зависимость величины угла резания $\delta$ от угла наклона винтовой канавки $\varepsilon$. С увеличением угла наклона винтовой канавки угол резания уменьшается.

Конструкция сверла определяется его технологическим назначением: видом обрабатываемого материала, направлением сверления относительно волокон древесины или плоскостей древесной плиты (в пласть или кромку) и др. Это связано с образованием и отводом стружки, силовыми и температурными напряжениями, которые, в свою очередь, зависят от угла резания $\delta$ и угла наклона винтовой канавки $\varepsilon$.

Углы резания в данной работе рассматриваются в плоскости движения, в данном случае в плоскости, нормальной к режущей кромке, следовательно, в зависимости от угла наклона режущей кромки к оси сверла $\varepsilon$ углы резания будут иметь различные значения, не совпадающие с контурными (за исключением случая для $\angle \varepsilon=90^{\circ}$ ). Кроме того, вследствие расположения канавки соответствующего профиля по винтовой линии углы резания приобретают различные значения по длине режущей кромки.

Ключевые слова: конструкция, совершенствование, угол резания, сверление, сверло.

Для цитирования: Машорипова Т. А., Гришкевич А. А. Особенности трансформации угла резания сверлильного инструмента в зависимости от положения его лезвия // Труды БГТУ. Сер. 1, Лесное хоз-во, природопользование и перераб. возобновляемых ресурсов. 2021. № 2 (246). C. 327-332.

\author{
T. A. Mashoripova, A. A. Grishkevich \\ Belarusian State Technological University
}

\section{FEATURES OF TRANSFORMATION OF THE CUTTING ANGLE OF THE DRILLING TOOL DEPENDING ON THE POSITION OF ITS BLADE}

The article describes a theoretical study of the influence of the angle of inclination of the blade to the axis of the screw drill on the cutting angle. In this paper, the dependence of the value of the cutting angle $\delta$ on the angle of inclination of the helical groove $\varepsilon$ is established. As the angle of inclination of the screw groove increases, the cutting angle decreases.

The design of the drill is determined by its technological purpose: the type of material to be processed, the direction of drilling relative to the wood fibers or the planes of the wood plate (in the layer or edge), and more. This is due to the formation and removal of chips, force and temperature stresses, which, in turn, depend on the cutting angle $\delta$ and the angle of inclination of the helical groove $\varepsilon$.

The cutting angles, in this paper, are considered in the plane of motion, in this case in the plane normal to the cutting edge, therefore, depending on the angle of inclination of the cutting edge to the drill axis, the cutting angles will have different values that do not coincide with the contour ones (except for the case for $\angle \varepsilon=90^{\circ}$ ). In addition, due to the location of the groove of the corresponding profile along the helix, the cutting angles acquire different values along the length of the cutting edge.

Key words: design, improvement, cutting angle, drilling, drill.

For citation: Mashoripova T. A., Grishkevich A. A. Features of transformation of the cutting angle of the drilling tool depending on the position of its blade. Proceedings of BSTU, issue 1, Forestry. Nature Management. Processing of Renewable Resources, 2021, no. 2 (246), pp. 327-332 (In Russian).

Введение. Сверление - один из очень распространенных методов механической обработки древесных материалов с целью получения отверстий. Формы и конструкции современного инструмента для обработки отверстий достигли значительного развития в связи с многочисленными технологическими задачами различных направлений деревообрабатывающей промышленности. И все же наиболее широко применяются винтовые сверла. Они представляют собой довольно сложный режущий инструмент. Работает он в более тяжелых условиях по сравнению с фрезерным, пильным и токарным инструментами. Теоретические исследования 
всегда являются приближенными и не учитывают всех факторов, которые влияют на искомую величину [1].

Как правило, экспериментальное решение инженерных задач сводится к нахождению закономерностей влияния входных переменных факторов процесса на выходные показатели. Полученные закономерности позволяют оценить воздействие входных переменных, что дает возможность в дальнейшем управлять технологическим процессом, делая его оптимальным.

Обработка древесины и древесных материалов методом сверления остается значимым технологическим процессом в столярно-строительном и мебельном производствах. Существуют различные методики проведения экспериментов по изучению свойств дереворежущего инструмента. Но большинство из них охватывает не более одного исследуемого варьируемого параметра, влияющего на интересующий нас показатель - качество обработанной поверхности [2].

Важными характеристиками сверлильного инструмента, влияющими на качество обработки, силы и мощность резания, являются угол наклона винтовой канавки $\varepsilon$, угол подъема винтовой канавки $\tau$, диаметр сверла $D$ и угол при вершине $2 \varphi$ (рис. 1).

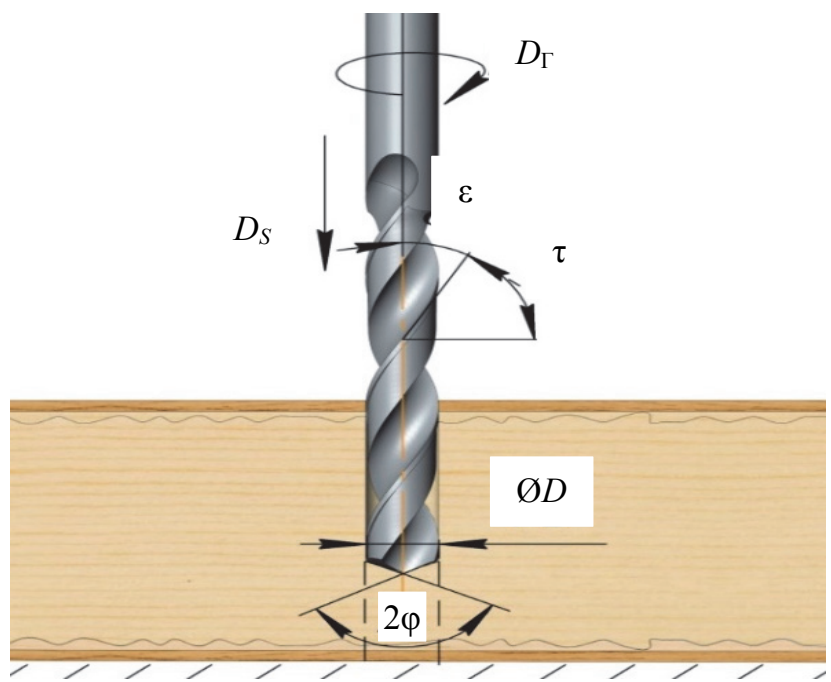

Рис. 1. Схема обработки сверлением

На процесс резания древесины и древесных материалов методом сверления оказывают влияние много факторов. Можно выделить три основные группы: 1) факторы, относящиеся к исследуемому материалу (физико-механические свойства материалов; 2) факторы, относящиеся к режущему инструменту (геометрические параметры сверла, углы резания и др.); 3) факторы, относящиеся к режимам эксплуатации инструмента, такие как скорость резания или обработки (скорость главного движения, скорость подачи) [3].
Таким образом цель исследования - изучение особенностей трансформации угла резания в зависимости от изменения положения лезвия сверлильного инструмента.

Задачи исследования:

1) определить конструкцию сверла, наиболее часто используемого в производстве;

2) установить значимый угол резания, влияющий на силовые характеристики процесса сверления.

Объект исследования - сверло. Предмет исследования - трансформация угла резания.

Основная часть. При сверлении древесных материалов наиболее распространенной конфигурацией режущей части сверла являются коническая заточка и форма с подрезателями и направляющим центром. При этом при второй форме сверло имеет четыре режущих элемента: две главные режущие кромки и два подрезателя. Гораздо реже применяют коническую заточку, когда сверло имеет две режущие кромки, наклоненные под углом $\varphi$ к оси вращения. В данной работе мы будем рассматривать сверла с конической заточкой, так как у сверл с центром и подрезателями угол наклона витовой канавки, как правило, $90^{\circ}$ [4-10].

Наибольшее влияние на усилие резания оказывают угловые параметры главной режущей кромки - угол резания $\delta$ и задний угол $\alpha$. Направляющий центр, назначение которого повысить точность сверления, и подрезатели, улучшающие качество сверления, характеризуются в основном расстоянием над главной режущей кромкой. Изменение углов резания главной режущей кромки оказывает влияние только на силовые показатели процесса сверления, так как качество сверления зависит только от боковых режущих элементов сверла [3, 11, 12-15].

Как известно, углы резания связаны и зависят от плоскости движения и плоскости, нормальной к режущей кромке. В зависимости от угла наклона режущей кромки к оси сверла $\varepsilon$ углы резания будут иметь различные значения. Они не будут совпадать с контурными, за исключением случая, когда $\angle \varepsilon=90^{\circ}$. Кроме того, вследствие расположения канавки соответствующего профиля по винтовой линии углы резания приобретают различные значения по длине режущей кромки [13].

Известно, что углы наклона $\varepsilon$ и подъема $\tau$ винтовой канавки зависят от шага последней и обусловливаются следующими соотношениями:

$$
\begin{aligned}
& \operatorname{tg} \tau=\frac{H}{2 \pi R} \\
& \operatorname{tg} \varepsilon=\frac{2 \pi R}{H},
\end{aligned}
$$


где $H$ - шаг винтовой канавки, мм; $R$ - радиус сверления, мм; $\varepsilon-$ угол наклона винтовой канавки, град.

Следовательно, при данном шаге винтовой канавки $H$, определяющем для периферийной точки режущей кромки значения углов $\varepsilon$ и $\tau$, значения углов в плоскости сечения, параллельной оси, для точек режущей кромки, близлежащих к оси сверла, будут иные - значение угла наклона $\varepsilon$ будет уменьшаться, а углы подъема $\tau$ увеличиваться. Это имеет отношение и для значений углов резания.

Аналитическая зависимость углов резания и контурных углов винтовой канавки может быть определена графическим способом приведенным ниже.

На рис. 1 представлено спиральное сверло с конической заточкой, с углом подъема винтовой канавки $\tau$ и углом при вершине $\varphi=2 \cdot \varepsilon$.

Для определения угла $\delta$ ' строим на режущей кромке ряд плоскостей, образующих параллелепипед, где $K$ - плоскость, касательная к конической поверхности, описываемой режущей кромкой при вращении; $S$ - плоскость, касательная к цилиндрической поверхности сверла; $R$ - плоскость (осевая), проходящая через ось сверла и режущую кромку; $H$ - плоскость, касательная к передней поверхности (по винтовой канавке) сверла в точке $a ; N$ - плоскость, перпендикулярная к режущей кромке в точке $a$.
Образованный данными плоскостями параллелепипед заключает в себя следующие углы: $\angle a b c, \angle b c d, \angle b a c$ в прямоугольных треугольниках $\triangle a b c, \triangle a b d, \triangle b c d$.

$$
\angle b d c=-\frac{\varphi}{2}=\varepsilon,
$$

где $\varphi$ - угол при вершине, град; $\varepsilon-$ половина угла при вершине, град. (Он равен $\omega)$.

$$
\angle b a d=\angle \tau,
$$

где $\tau$ - угол подъема винтовой канавки, град.

$$
\angle b a c=\delta^{\prime}
$$

где $\delta^{\prime}-$ угол резания, град.

Из прямоугольных треугольников $\triangle a b c$, $\triangle a b d, \triangle b c d$ находим зависимость между углами $\delta^{\prime}$ и $\tau$.

Из треугольника $\triangle a b c$ следует, что

$$
\operatorname{tg} \psi=\frac{b c}{a b} .
$$

Из треугольника $\triangle a b d$ следует, что

$$
a b=\frac{d b}{\operatorname{tg} \tau} .
$$

Из треугольника $\triangle b c d$ следует, что

$$
b c=d b \cdot \sin \varepsilon .
$$

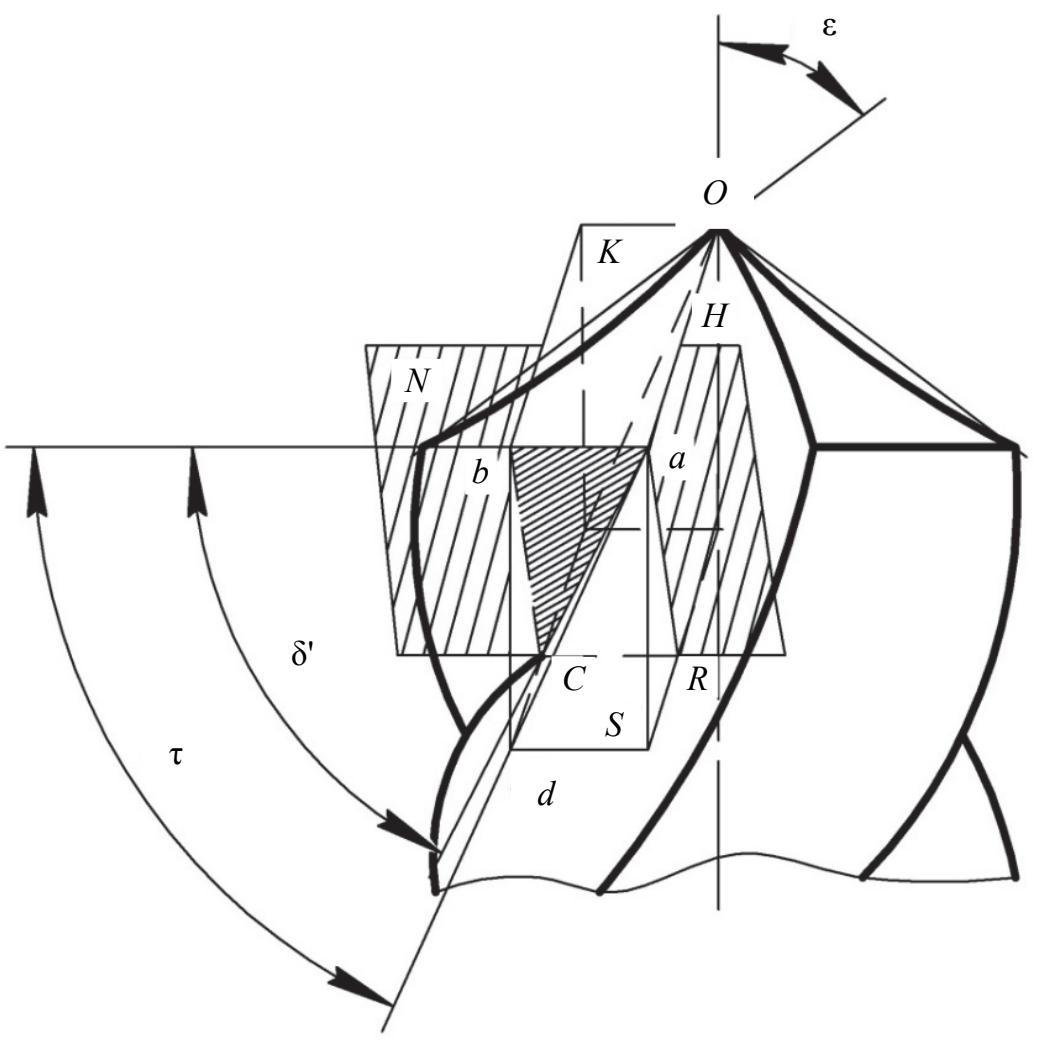

Рис. 1. Графическое изображение контурных и пространственных углов резания 


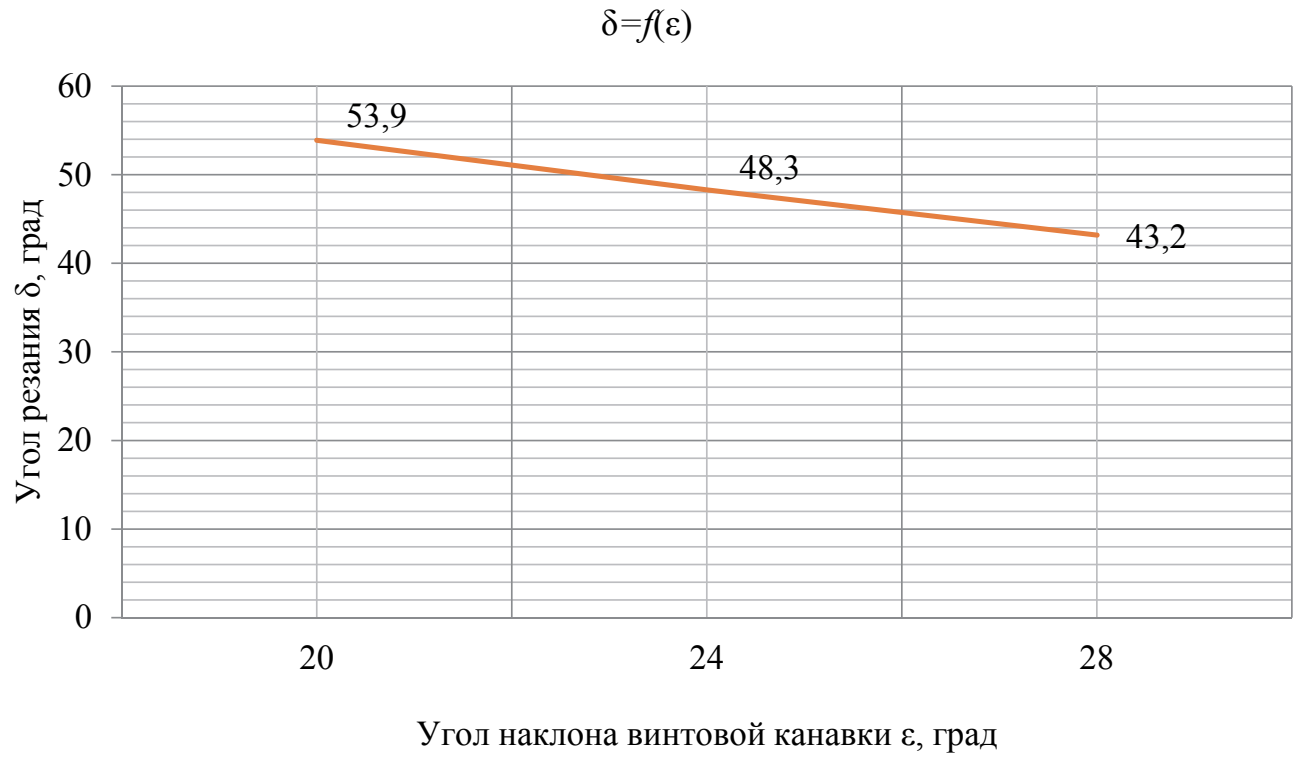

Рис. 2. График зависимости угла резания от угла наклона винтовой канавки $\varepsilon$

Из формул (3)-(8) получаются следующие зависимости:

$$
\begin{gathered}
\operatorname{tg} \delta^{\prime}=\frac{d b \cdot \sin \varepsilon}{d b} \cdot \operatorname{tg} \tau \\
\operatorname{tg} \delta^{\prime}=\sin \varepsilon \cdot \operatorname{tg} \tau
\end{gathered}
$$

На основе формул (1) и (2) можно сделать следующий вывод:

$$
\operatorname{tg} \tau=(\operatorname{tg} \varepsilon)^{-1}=\operatorname{ctg} \varepsilon .
$$

Формула (9) примет вид

$$
\operatorname{tg} \delta^{\prime}=\sin \omega \cdot \operatorname{ctg} \varepsilon .
$$

Угол резания составит

$$
\delta^{\prime}=\operatorname{arctg}(\sin \varepsilon \cdot \operatorname{tg} \tau) .
$$

Для сверл малого диаметра (в данной работе сверло $D=10$ мм) угол наклона винтовой канавки составляет $\varepsilon=20-28^{\circ}$. Для построения графика возьмем три значения угла наклона винтовой канавки $\varepsilon=20^{\circ}, \varepsilon=24^{\circ}$ и $\varepsilon=28^{\circ}$. Половина угла при вершине принята из конструкции сверла $\omega=30^{\circ}$ и величина постоянна.

$$
\begin{aligned}
& \delta_{1}^{\prime}=\operatorname{arctg}(\sin 30 \cdot \operatorname{ctg} 20)=53,9^{\circ} ; \\
& \delta_{2}{ }_{2}=\operatorname{arctg}(\sin 30 \cdot \operatorname{ctg} 24)=48,3^{\circ} ; \\
& \delta^{\prime}{ }_{3}=\operatorname{arctg}(\sin 30 \cdot \operatorname{ctg} 28)=43,2^{\circ} .
\end{aligned}
$$

На основании этих данных построим график. На рис. 2 представлен график зависимости угла резания $\delta$, град, от угла наклона винтовой канавки $\varepsilon$, град.

Из графика можно сделать следующий вывод: при увеличении угла наклона винтовой канавки $\varepsilon$, град, угол резания $\delta$, град, уменьшается. Также с уменьшением угла резания уменьшаются крутящий момент $M_{\text {ок, }} \mathrm{H} \cdot \mathrm{M}$, и осевое усилие $F_{0}, \mathrm{H}$.

Заключение. В данной работе установлена зависимость угла резания $\delta$ от угла наклона режущей кромки лезвия инструмента. С увеличением угла наклона лезвия угол резания уменьшается.

С уменьшением угла резания уменьшаются крутящий момент $M_{\text {ок, }} \mathrm{H} \cdot$ м, и осевое усилие $F_{0}, \mathrm{H}$, вследствие чего улучшается качество получаемой поверхности при сверлении.

Таким образом, определена трансформация угла резания в зависимости от изменения угла наклона винтовой канавки. С незначительным изменением положения лезвия сверлильного инструмента статический угол резания уменьшается на 5 град. Особенностью трансформации угла резания сверлильного инструмента в зависимости от положения его лезвия является большое изменение угла $\delta$, а именно с $20^{\circ}$ до $24^{\circ}$ изменение происходит почти на $11 \%$ (5,6 град), a с $24^{\circ}$ до $28^{\circ}$ более чем на $10 \%$ (5,1 град). Из этого следует, что при выборе конструкции сверла следует учитывать данный фактор при установлении технологических режимов и при выборе таких угловых параметров, как задний угол $\alpha$ и угол резания $\delta$.

\section{Список литературы}

1. Глебов И. Т., Мартинон А. Сверление древесины: учеб.-справ. пособие. Екатеринбург: УГЛТУ, 2016. $54 \mathrm{c}$. 
2. Бершадский А. Л. Расчет режимов резания древесины. Минск: Вышэйшая школа, 1966. 176 с.

3. Цуканов Ю. А., Амалицкий В. В. Обработка резанием древесностружечных плит. М.: Лесная пром-сть, 1966. $94 \mathrm{c.}$

4. Сверлильный инструмент. URL: https://www.leuco.com/RU/RU/Boring_Bits (дата обращения: 22.03.2021).

5. Сверла. URL: https://leitz.by/index.pl?act=SECTION\&section=sverla (дата обращения: 22.03.2021).

6. Сверла. URL: https://faba78.ru/products/category/sverla (дата обращения: 22.03.2021).

7. Сверла для присадочных станков. URL: http://freud-catalog.ru/catalog/376/692/index.htm (дата обращения 22.03.2021).

8. Сверла для деревообработки. URL: https://www.stanki.by/catalog/svyerla_/ (дата обращения 22.03.2021).

9. Сверла спиральные дереворежущие. Технические условия: ГОСТ 22057-76. Введ. 01.01.1978. М.: Изд-во стандартов, 1978. 21 с.

10. Сверла спиральные с цилиндрическим хвостовиком. Технические условия: ГОСТ 10902-77*. Введ. 01.01.1979. М.: Изд-во стандартов, 1979. 23 с.

11. Любченко В. И. Резание древесины и древесных материалов: учеб. пособие. М.: Лесная промсть, 1986. 296 с.

12. Аникеенко А. Ф., Гришкевич А. А., Гаранин В. Н. Влияние элементов режима сверления ламинированных древесностружечных плит на качество поверхности // Труды БГТУ. Сер. 1, Лесное хоз-во, природопользование и перераб. возобновляемых ресурсов. 2017. № 2 (198). C. 391-394.

13. Грубе А. Э. Дереворежущие инструменты: учеб. пособие. М.: Лесная пром-сть, 1971. 344 с.

14. Глебов И. Т. Обработка древесины на станке с ЧПУ: учеб. пособие. Екатеринбург: УГЛТУ, 2018. $142 \mathrm{c}$.

15. Морозов В. Г. Дереворежущий инструмент: справочник. М.: Лесная пром-сть, 1988. 344 с.

\section{References}

1. Glebov I. T., Martinon A. Sverleniye drevesiny [Drilling wood]. Ekaterinburg, UGLTU Publ., 2016. 54 p.

2. Bershadski A. L. Raschet rezhimov rezaniya drevesiny [Computation of cutting wood]. Minsk, Vysheyshaya shkola Publ., 1966. 176 p.

3. Tsukanov Yu. A., Amalitskiy V. V. Obrabotka rezaniyem drevesnostruzhechnykh plit [Chipboard cutting]. Moscow, Lesnaya promyshlennost' Publ., 1966. 94 p.

4. Sverlil'nyy instrument [Drilling tools]. Available at: https://www.leuco.com/RU/RU/Boring_Bits (accessed 22.03.2021).

5. Sverla [Drills]. Available at: https://leitz.by/index.pl?act=SECTION\&section=sverla (accessed 22.03.2021).

6. Sverla [Drills]. Available at: https://faba78.ru/products/category/sverla (accessed 22.03.2021).

7. Sverla dlya prisadochnyh stankov [Drill bits for filler machines]. Available at: http://freud-cata$\log$.ru/catalog/376/692/index.htm (accessed 22.03.2021).

8. Sverla dlya derevoobrabotki [Drills for woodworking]. Available at: https://www.stanki.by/cata$\log /$ svyerla/ (accessed 22.03.2021).

9. GOST 22057-76. Sverla spiral'nyye derevorezhushchiye. Tekhnicheskiye usloviya [Wood-cutting spiral drills. Specifications]. Moscow, Izdatel'stvo standartov Publ., 1978. 21 p. (In Russian).

10. GOST 10902-77*. Sverla spiral'nyye s tsilindricheskim khvostovikom. Tekhnicheskiye usloviya [Spiral drills with a cylindrical shank. Specifications]. Moscow, Izdatel'stvo standartov Publ., 1977. 23 p. (In Russian).

11. Lyubchenko V. I. Rezaniye drevesiny $i$ drevesnykh materialov [Cutting wood and wood material]. Moscow, Lesnaya promyshlennost' Publ., 1986. 296 p.

12. Anikeenko A. F., Grishkevich A. A., Garanin V. N. The influence of the elements of the mode of drilling laminated chipboard on the surface quality. Trudy BGTU [Proseedings of BSTU], issue 1, Forestry. Nature Management. Processing of Renewable Resources, 2017, no. 2 (198), pp. 391-394 (In Russian).

13. Grube A. E. Derevorezhushchiye instrumenty [Woodworking tools]. Moscow, Lesnaya promyshlennost' Publ., 1971. 344 p.

14. Glebov I. T. Obrabotka drevesiny na stanke s CHPU [Wood processing on a CNC machine]. Yekaterinburg, UGLTU Publ., 2018. 142 p.

15. Morozov V. G. Derevorezhushchiy instrument: spravochnik [Woodworking tools: Reference]. Moscow, Lesnaya promyshlennost' Publ., 1988. 344 p. 


\section{Информация об авторах}

Машорипова Татьяна Александровна - аспирант кафедры деревообрабатывающих станков и инструментов. Белорусский государственный технологический университет $(220006$, г. Минск, ул. Свердлова, 13а, Республика Беларусь). E-mail: t.a.mashoripova@mail.ru

Гришкевич Александр Александрович - кандидат технических наук, доцент, заведующий кафедрой деревообрабатывающих станков и инструментов. Белорусский государственный технологический университет (220006, г. Минск, ул. Свердлова, 13a, Республика Беларусь). E-mail: dosy@belstu.by

\section{Information about the authors}

Mashoripova Tatiana Aleksandrovna - $\mathrm{PhD}$ student, the Department of Woodworking Machines and Tools. Belarusian State Technological University (13a, Sverdlova str., 220006, Minsk, Republic of Belarus). E-mail: t.a.mashoripova@mail.ru

Grishkevich Aleksandr Aleksandrovich - PhD (Engineering), Associate Professor, Head of the Department of Woodworking Machines and Tools. Belarusian State Technological University (13a, Sverdlova str., 220006, Minsk, Republic of Belarus). E-mail: dosy@belstu.by 